\title{
Relações Familiares de Adolescentes Cumprindo Medida Socioeducativa Restritiva de Liberdade: Uma Revisão Narrativa da Literatura
}

\author{
Angélica Maria Ferreira de Melo Castro* \\ Universidade Federal Rural de Pernambuco, Recife, Pernambuco, Brasil \\ Maycoln Leôni Martins Teodoro \\ Programa de Pós-Graduação em Psicologia da Universidade Federal de Minas Gerais, \\ Belo Horizonte, Minas Gerais, Brasil
}

\begin{abstract}
Resumo
O objetivo deste artigo foi realizar uma revisão narrativa sobre a relação entre o sistema familiar e a conduta infracional de jovens. Foram consultadas as bases de dados SciELO; LILACS; BVS; PePSIC; PsycNET; APA PsycNET; Science Direct e Web of Knowledge (ISI). Resultaram da busca 19 artigos que atenderam aos critérios de inclusão. A análise dos artigos mostrou que alguns fatores familiares comumente encontram-se associados à condição de infrações à lei e ao cumprimento de medida sócioeducativa de internação pelos adolescentes. São eles: os sócio-econômicos, a estrutura familiar, a violência intrafamiliar, as práticas educacionais e monitoramento parental, a fragilidade dos vínculos familiares, os familiares em conflito com a lei e os fatores biológicos. Assim, pode-se constatar que a família, ao não cumprir sua função de proteção, passa a ser considerada um fator de risco ao bem-estar de muitas crianças e adolescentes.
\end{abstract}

Palavras-chave: Relações familiares, violência, adolescentes em conflito com a lei.

\section{Family Relationships of Adolescents under Socio-Constrained Freedom: A Systematic Narrative Review}

\begin{abstract}
The purpose of this article was to realize a narrative review of the relation between the family system and the criminal conduct of adolescents. There were used the data bases: SciELO; LILACS; BVS; PePSIC; PsycNET; APA PsycNET; Science Direct and Web of knowledge (ISI). The search resulted on 19 articles that complied the inclusion criteria. The articles' analysis showed that some family factors are commonly found associated with law infraction and fulfillment of social educative freedom restriction measures by the adolescents. They are: socioeconomics level, family structure, domestic violence; educational practices and parental control, the frailty of family bonds, family members in conflict with the law, and biologic factors. So, it could be stated that when the family doesn't fulfill its protective function, it starts to be seen as a risk factor to the well-being of many children and adolescents.
\end{abstract}

Keywords: Family relationships, violence; adolescents in conflict with the law.

Endereço para correspondência: Rua Sargento Silvino de Macêdo, 155, Apto. 302 - B, Bairro Imbiribeira, Recife, PE, Brasil 51160-060. E-mail: angelicamfmcastro@gmail.com e mlmteodoro@hotmail.com 


\section{Relaciones Familiares de los Adolescentes em la Internación Socioeducativa: Una Revisión Narrativa de la Literatura}

\section{Resumen}

El propósito de este artículo es realizar una revisión narrativa de la relación entre el sistema familiar y el comportamiento de los jóvenes delito. Fueron consultadas las bases de datos SciELO; LILACS; BVS; PePSIC; PsycNET; APA PsycNET; Science Direct y Web of Knowledge (ISI). La búsqueda resultó en 19 artículos que cumplían los criterios de inclusión. El análisis de los artículos mostraron que algunos factores familiares son comúnmente asociados con la condición de violaciónes de la ley y el cumplimiento de castigo socioeducativo de internación. Son estos factores: la estructura socioeconómica de la familia, la violencia doméstica, las prácticas educativas y de supervisión parental, la fragilidad de los lazos familiares, parientes en conflicto con la ley, y factores biológicos. Así, se puede ver que la familia a no cumplir su función protectora, es considera un factor de riesgo para el bienestar de los niños, niñas y adolescentes.

Palabras clave: Las relaciones familiares, violencia, los adolescentes en conflicto con la ley.

Os atos infracionais cometidos por crianças e adolescentes vêm sendo amplamente divulgados pelos meios de comunicação. Estima-se que cerca de 20 mil adolescentes estavam em internação no fim de 2010 no Brasil cumprindo pena de restrição de liberdade por terem praticado algum tipo de crime, apresentando um crescimento de $4,5 \%$ desta população entre os anos 2009 e 2010 (Secretaria Nacional de Promoção dos Direitos das Crianças e dos Adolescentes [SNPDCA], 2011). Estes dados preocupam as esferas sociais e revelam que políticas públicas devem ser discutidas e repensadas, com vistas a amenizar o crescimento deste problema social.

Conhecer e compreender os elementos e/ou variáveis que podem estar relacionados ao fato de crianças e adolescentes realizarem comportamentos que culminam em situações de conflito com a lei torna-se imprescindível, uma vez que viabiliza a fomentação de projetos de intervenção e de prevenção que auxiliem na diminuição de comportamentos de transgressões à lei. Neste sentido, pesquisadores vêm estudando a existência de fatores de riscos que podem propiciar aos adolescentes situações de conflito com a lei, tais como: uso de drogas, violência familiar, personalidades, dentre outros (Christiansen \& Knussmann, 1987; Galo \& Willians, 2005; Pacheco \& Hutz, 2009; Webster-Stratton, 1998).
Galo e Willians (2005) revisaram as principais publicações científicas entre 1997 a 2003 e apontaram que dificuldades de aprendizagem e baixo rendimento escolar, problemas na família, problemas no meio social, consumo de drogas e baixo poder econômico estavam entre os principais fatores de risco para crianças e adolescentes apresentarem conflito com a lei. Entretanto, os autores acrescentam que não seria a simples existência dos fatores de risco um fator determinante à prática infracional da população infanto-juvenil, pois o comportamento humano não pode ser predito por meio de conjunto de variáveis isoladas, mas sim como um processo multideterminado por relações complexas entre as diferenças individuais e sócio-culturais.

Dentre os fatores elencados, o ambiente familiar possui extrema importância por ser o local de socialização primária do homem, pois, segundo Pacheco e Hutz (2009), 53\% da variância do comportamento infrator pode ser explicada por algumas característica familiares, tais como: número de irmãos, práticas educativas parentais, conflitos na família. Deste modo, é necessário um aprofundamento das relações entre os aspectos familiares e o comportamento conflitivo com a lei. Sabe-se que conflitos nas relações conjugais podem provocar prejuízos ao desenvolvimento da criança e do adolescente, 
não sendo benéfico para crianças vivenciarem, dentro do ambiente familiar, situação de agressão física, submissão, agressão ou hostilidade verbal, isolamento, episódios de perseguição e ameaças que comprometem a união da família, bem como ocasionem em situações de exposição das crianças a conflitos (Davies, Myers, Cummings, \& Heindel, 1999).

Assim, considerando a importância da família para a socialização e formação do indivíduo, o objetivo deste artigo será realizar uma revisão narrativa de estudos sobre a relação entre o sistema familiar e a conduta infracional de jovens, segundo uma síntese da literatura científica vigente. Ou seja, a partir de informações mais contextualizadas com os dias atuais, com o intuito de investigar quais aspectos familiares podem contribuir para a formação de conduta infracional de seus membros na contemporaneidade, haja vista a lacuna de material existente.

\section{Método}

\section{Bases e Estratégias de Busca}

A busca dos artigos foi realizada por dois juízes em sete bases de dados: SciELO, LILACS, BVS, PePSIC, PsycNET, APA PsycNET, Science Direct e Web of Knowledge (ISI), no período compreendido entre os dias 23 de janeiro a 6 de dezembro de 2012, restringindo-se aos artigos de línguas inglesa e portuguesa publicados a partir de 2005 até 2012.

Os descritores utilizados foram relações familiares e conflito com a lei (family relationships and conflict with the law), família e conflito com a lei (family and conflict with the law) e adolescentes infratores e família (family and adolescent offender). $\mathrm{O}$ campo selecionado a se pesquisar foi "assunto" ou "palavra", quando a opção assunto era inexistente. Fez-se uso do operador boleano "and", não recorrendo a truncaturas.

\section{Critérios de Seleção dos Artigos e Extração dos Dados}

Os artigos incluídos nessa revisão narrativa inicialmente deveriam preencher alguns requi- sitos a partir da leitura de seus resumos. Foram selecionados os seguintes critérios de inclusão: (a) Métodos Quantitativos; (b) Amostras compostas por adolescentes em conflito com a lei; (c) Investigação dessa amostra com a variável família e (d) Investigação de alguma relação familiar.

Em um segundo momento, foram excluídos artigos que analisavam a eficácia do sistema de internação de medidas sócio-educativas, a reinserção social dos adolescentes, fatores de reincidência dos atos infracionais, assim como os artigos que apresentavam a forma de editoriais, projetos e entrevistas. Ou seja, foram utilizados critérios de exclusão mais específicos.

Nesta fase, por se tratar de uma busca mais criteriosa, quando os resumos não foram esclarecedores, buscou-se ler o artigo na íntegra (texto completo) para evitar a possibilidade de deixar estudos importantes fora da revisão narrativa.

Os artigos que atenderam aos critérios de inclusão foram analisados e registrados pelos juízes em um protocolo elaborado pelos pesquisadores, que continha um resumo acrescido de comentários. Os resumos foram organizados de forma a apresentar a sua estrutura em tópicos, compostos por: título, resumo, introdução, objetivos, delineamento do estudo, amostra, instrumentos de coleta de dados e resultados de discussão.

\section{Resultados}

A partir dos descritores utilizados e dos critérios de inclusão e exclusão iniciais descritos na sessão anterior, foram encontrados 58 artigos potencialmente relevantes nas bases de dados após a leitura dos resumos disponíveis. Dois artigos eram repetidos, totalizando $56 \mathrm{pu}-$ blicações. Em seguida, após uma segunda leitura do material selecionado e a aplicação dos critérios de seleção mais específicos dos artigos, foram excluídos 25 artigos, como mostra a Figura 1. 
Fase 1: Definição

das bases de dados

Fase 2: Definição

dos descritores
Base de dados: Scielo, LILACS, BVS, PePSiC, PsycNET, APA PsycNET, Science Direct $\mathrm{e}$ Web of Knowedge (ISI)
34 artigos excluídos

- Amostra mista $(n=17)$

- Estudos qualitativos $(n=4)$

- Visaram a eficiência da medida socioeducativa $(n=7)$

- Objetivaram a socialização fora do

contexto familiar $(n=5)$

- Estudos de intervenção $(n=1)$

- Mesma publicação em duas bases

diferentes $(n=2)$

Fase 4: Análise dos textos completos
22 artigos analisados integralmente

3 artigos excluídos

- Validação de instrumentos $(n=2)$

- Adolescentes em liberdade assistida $(n=1)$

19 artigos incluídos na revisão

Figura 1. Esquema representativo dos procedimentos de seleção dos artigos.

Dos artigos encontrados, foram retirados aqueles que: (a) objetivaram avaliar a eficiência das medidas sócio-educativas e a importância da família no processo sócio-educativo $(n=7)$; (b) eram estudos qualitativos que não exploravam os fatores preditores e fatores de risco $(n=5)$; (c) eram compostos por amostra mista: constituída por familiares $(n=3)$; (d) eram compostos por amostra mista: constituída por menores e maiores de idade ( $n=2)$; (e) eram compostos por amostra mista, constituída por funcionários das instituições $(n=4)$; (f) focaram a socialização de forma geral e não a familiar $(n=5)$, $(\mathrm{g})$ eram compostos por amostra mista: adolescentes "delinquentes/ problemáticos" e menores em conflito com a lei $(n=7)$ e (h) objetivou o estudo de intervenções $(n=1)$.

A retirada destes artigos possibilitou uma análise composta por um material bibliográfico mais homogêneo. É importante ressaltar que, no caso dos artigos com amostra mista, os dados foram analisados de forma conjunta, ou seja, não tinham dados específicos para a população de adolescentes em conflito com a lei. Sendo apenas por esse motivo que os artigos compostos por amostra mista foram excluídos desta revisão.

Assim, 22 artigos foram selecionados para serem analisados criticamente e incluídos na revisão. Desses artigos, duas publicações foram excluídas por não terem como objetivo específico analisar a amostra de acordo com as relações familiares, pois visavam validar instrumentos e detectar psicopatologias. Por último, excluiu-se um artigo que era composto por amostra mista de adolescentes em liberdade condicional e com restrição total de liberdade. Dos 58 artigos, restaram 19, que foram selecionados nesta etapa e assim constituem o corpus desta revisão. 
Estes artigos estão descritos na Tabela 1, bem como os fatores que, segundo os estudos, encontram-se associados à condição de cumpri- mento de medida sócio-educativa de restrição de liberdade relacionada com a família. Os principais fatores de risco encontrados nestes artigos serão descritos nas próximas seções.

\section{Tabela 1}

Fatores Familiares Associados à Condição de Cumprimento de Medida Socioeducativa de Restrição de Liberdade

Autores, ano e local

Lima, Alcântara, Almeida, \& Alves (2006), Brasil.

Branco \& Wagner (2009), Brasil.

Krischer \& Sevecke (2008), EUA.

Gold, Sullivan, \& Lewis (2011), EUA.

Grogan-Kaylor, Ruffolo, Ortega, \& Clarke

(2008), EUA.

Nijhof, de Kemp, \& Engels (2009), Holanda.

Mack, Leiber, Featherstone, \& Monserud

(2007), EUA.

Carvalho \& Gomide (2005), Brasil.

Pacheco \& Hutz (2009), Brasil.

Ozen, Ece, Oto, Tirasci, \& Goren (2005), Turquia.

Caldwell, Beutler, Ross, \& Silver (2006), México.

Tia, Merry, \& Suyeon (2011), EUA.

Childs, Sullivan, \& Gulledge (2011), EUA.

Rodway et al. (2011), Inglaterra.

Wong (2011), Canada.

Carter, Fortson, \& Dusten (2006), EUA.

Kierkus \& Hewitt (2005), Canada.

Day, Leech, \& Goldschmidt (2011), EUA.

Nardi \& Dell`Aglio (2012), Brasil.
Principais fatores de risco associado à família

Violência intrafamiliar

Violência intrafamiliar; vínculos e conflitos familiares

Violência intrafamiliar

Violência intrafamiliar

Violência intrafamiliar

Violência intrafamiliar

Violência intrafamiliar

Práticas educacionais e monitoramento

Práticas educacionais e monitoramento

Práticas educacionais e monitoramento; condições socioeconômicas; estrutura familiar; parentes em conflito com a lei;

Práticas educacionais e monitoramento;

Práticas educacionais e monitoramento; parentes em conflito com a lei

Práticas educacionais e monitoramento

Vínculos e conflitos familiares

Condições socioeconômicas; estrutura familiar

Condições socioeconômicas

Estrutura familiar

Fatores genéticos

Estrutura familiar; vínculos e conflitos familiares; práticas educacionais

e monitoramento

\section{Fatores Sócio-econômicos e Educacionais}

Três estudos $(16,67 \%)$ revelaram as condições sócio-econômicas como um dos fatores que pode vir a caracterizar a família como um fator de risco ao desenvolvimento da conduta criminal dos adolescentes. A maioria das famílias, dos menores em cumprimento de medida sócio-educativa de restrição de liberdade, apresentou nos estudos níveis sócio-econômicos baixos, carac- 
terizados assim por condições de pobreza, exclusão e falta de amparo social (Kierkus \& Hewitt, 2005; Ozen et al., 2005; Wong, 2011).

A maioria das famílias, além de apresentar reduzido nível econômico, também apresentou baixos níveis educacionais concatenados com a grande quantidade de integrantes. Ou seja, por se tratar de família com grande número de membros (filhos) e pouca renda familiar, o nível econômico é reduzido, bem como o monitoramento das atividades de seus constituintes (Kierkus \& Hewitt, 2005).

Um dos estudos revelou que a maioria das crianças tinha crescido em um ambiente familiar composto por muitos integrantes e que o número médio dos irmãos era de 7,4. A probabilidade de cometer algum delito foi de 3 a 4 vezes maior em uma pessoa que tem 5-6 irmãos e 15 vezes maior em um indivíduo que tem 10 ou mais irmãos (Ozen et al., 2005). Neste sentido, observa-se que em alguns casos o nível sócio-econômico está relacionado com a estrutura familiar.

\section{Estrutura Familiar}

Quatro estudos $(22,22 \%)$ revelaram a estrutura familiar (forma de organização dos membros familiares e das relações estabelecidas entre si) como um dos fatores que pode vir a caracterizar este núcleo de socialização primária como um fator de risco à conduta criminal dos adolescentes. A maioria das famílias, que possui menores infratores desprovidos de liberdade, não é nuclear devido à separação dos pais, ao abandono do lar familiar, ao falecimento etc. Dessa forma, o outro membro constituiu um novo núcleo familiar ao ter um novo companheiro e novos filhos advindos desta união (Kierkus \& Hewitt, 2005; Nardi \& Dell Aglio, 2012; Ozen et al., 2005; Wong, 2011).

Wong (2011), em uma análise sobre a estrutura da família, observou que esta característica não interage com o sexo, com o nível sócio-econômico ou com a localidade da residência. Dessa forma, após analisar o tamanho da amostra e a sensibilidade dos testes estatísticos, este autor concluiu que os meninos, meninas, ricos, pobres, jovens que residem na cidade e os seus pais irão responder, em grande parte, da mesma maneira, ao crescerem e se desenvolverem em famílias não nucleares. Isto significa que, segundo esta pesquisa, eles constituirão parte do grupo de risco de adolescentes propensos a cometer atos infracionais (Wong, 2011).

\section{Violência Intrafamiliar}

Dentre os artigos analisados, observou-se que seis estudos $(33,33 \%)$ revelaram a violência intrafamiliar como uma das características que pode vir a definir a família como um fator de risco ao desenvolvimento da conduta criminal dos adolescentes. A maioria das famílias, dos menores em cumprimento de medidas sócio-educativas com restrição de liberdade, teve a experiência de violência familiar (Mack et al., 2007).

Segundo Lima et al. (2006), 92\% dos adolescentes infratores já experimentaram, pelo menos uma vez, algum tipo de violência no ambiente familiar. Dentre tal porcentagem, 89,6 foram violências materiais ou físicas. A parentalidade abusiva pode converter a culpabilidade a um terceiro e assim fomentar comportamentos de infrações à lei (Gold et al., 2011). Nesta direção, Nijhof et al. (2009) demonstram que a frequência com que adolescentes realizam atos de violência física está diretamente relacionada à frequência na qual o adolescente foi vítima de violência quando criança.

Resultados divergentes dos apresentados anteriormente foram encontrados em um estudo que buscou examinar a partir de diversas referências teóricas como é caracterizada a relação existente entre a estrutura familiar e a delinquência juvenil. Como resultado, observou-se, por meio de análises de regressão binomial, que as famílias violentas ou não violentas não foram um preditor significativo da delinquência da criança. Pelo contrário, o apego maternal emergiu como o mais importante determinante no comportamento delinquente entre os jovens de todos os tipos e estruturas de família, demonstrando que, quanto maior o apego, menor será a probabilidade de comportamentos de delinquência pelos adolescentes (Mack et al., 2007). 


\section{Práticas Educacionais e Monitoramento Parental}

Quanto às práticas educacionais e monitoramento parental, entendidas como estratégias disciplinares ultilizadas pelos pais objetivando a promoção comportamentos sociais moralmente desejáveis e redução dos inadequados, destaca-se que seis estudos $(33,33 \%)$ revelaram-nas como um dos elementos que vem a caracterizar a família como um fator de risco ao desenvolvimento da conduta criminal dos adolescentes. Em famílias de adolescentes em conflito com a lei, pode-se observar que o nível de estilo parental familiar (conjunto de atitudes, modelo parental e práticas educativas utilizadas pelos pais visando à promoção da socialização dos filhos) e o monitoramento familiar (filho, pai e mãe) não são positivos, ou seja, não propiciam um desenvolvimento pró-social como seria papel da família realizar (Carvalho \& Gomide, 2005; Nardi \& Dell Aglio, 2012; Ozen et al., 2005; Tia et al., 2011).

Em outro estudo, uma análise de regressão indicou que o comportamento antissocial de familiares, o número de irmãos, o uso de drogas pelo adolescente, os conflitos na família e as práticas educativas parentais explicaram 53\% da variância do comportamento infrator. Os resultados apontaram a importância da família no desenvolvimento da conduta infratora (Pacheco \& Hutz, 2009). Assim, a literatura referente aos processos familiares e aos atos infracionais revela que o monitoramento parental relaciona-se negativamente à delinquência (Caldwell et al., 2006). Acrescenta-se ainda que, através dos efeitos oriundos da modelagem, existe o risco dos pais influenciarem, de alguma forma, os filhos ao uso de substâncias químicas e à delinquência juvenil, por meio das práticas educacionais (Childs et al., 2011).

\section{Fragilidade dos Vínculos Familiares}

A fragilidade dos vínculos familiares (baixos níveis de afeto, pouca coesão familiar, etc) foi abordada por $22,22 \%$ dos artigos, isto é, quatro deles revelaram o quanto esta condição pode ser considerada um fator de risco ao desenvolvimento da conduta criminal dos adolescentes. Embora a família ainda seja a instituição que os jovens acreditam poder ajudá-los, esta se mostrou extremamente fragilizada, vulnerável e com pouca capacidade de atuação. Branco e Wagner (2009) consideraram que as médias de funcionamento familiar foram baixas, revelando que a família passa, em muitos momentos, a assumir um papel de fator de risco ao invés de um fator de proteção à conduta criminal de adolescentes.

Inúmeras pesquisas apontam para fatores de risco relacionados com a prática infracional. Os fatores mais frequentes são: as condições da família com baixos níveis de afeto, pouca coesão, indiferença generalizada e vínculo pouco afetivo nas relações interpessoais, além dos problemas familiares. De fato, estudos demonstram que conflitos familiares e disfunção familiar são fatores que podem acarretar em situações de comportamentos de atos infracionais por adolescentes (Nardi \& Dell Aglio, 2012; Rodway et al., 2011).

\section{Familiares em Conflito com a Lei}

Dentre os 18 artigos, três $(16,67 \%)$ revelaram que as ações de infrações à lei em adolescentes são maiores em adolescentes que têm membros familiares em conflito com a lei (Nijhof et al., 2009; Ozen et al., 2005; Tia et al., 2011). Em um estudo realizado com 577 adolescentes infratores, 163 apresentaram pelo menos um dos pais criminoso, 33 deles tinham a mãe e o pai considerados criminosos e 34 foram expostos à criminalidade dos pais quando crianças. Acrescenta-se ainda que, se ambos os pais eram criminosos, a criança apresentava uma maior frequência de realizar atos infracionais de violência, tornando claro que esses resultados oferecem uma melhor compreensão da influência que os pais, com um histórico criminal, exercem sobre seus filhos (Nijhof et al., 2009).

Em outro estudo, entre 165 jovens em medidas de privação de liberdade, $14,5 \%$ tinham um irmão delinquente. Então, ao encontrar uma associação moderadamente forte entre esses dois fatores, constata-se que o irmão que comete ou cometeu atos de infrações à lei pode influenciar 
irmãos adolescentes mais novos a também cometerem os mesmos delitos (Ozen et al., 2005).

Tia et al. (2011) analisaram adolescentes do sexo feminino e observaram que elas tendem a apresentar mais resistência para cometer atos infracionais quando não apresentam membros familiares com históricos de criminalidade. Assim, demonstra-se que realmente parece existir uma relação entre possuir membros familiares em conflito com a lei e cometer infrações a leis em amostras juvenis de ambos os sexos, feminino e masculino.

\section{Fatores Biológicos}

Por fim, apenas um estudo (5,3\%) apontou para o fato de alguns fatores biológicos durante a gestação serem considerados influenciadores no desenvolvimento da conduta criminal dos adolescentes. Day et al. (2011) investigaram a hipótese da existência de uma relação entre a exposição pré-natal à maconha e a realização de atos infracionais na adolescência de jovens por terem o desenvolvimento neurocognitivo do feto comprometido por causa da exposição à droga durante a gestação. Os autores encontraram que, aos 10 anos, havia uma tendência dos adolescentes a apresentarem sintomas depressivos e problemas de atenção. Já aos 14 anos, existia uma relação significativa com a exposição à maconha durante a gestação da mãe e comportamento delinquente.

\section{Discussão}

Este artigo de revisão compilou as investigações empíricas sobre as relações familiares e jovens em conflito com a lei. Como exposto, o conjunto de publicações revelou alguns fatores que, segundo as pesquisas, encontram-se associados à condição de cumprimento de medida sócio-educativa de restrição de liberdade relacionado com a família.

Dentre as categorias encontradas que constituem fatores de risco para o envolvimento dos adolescentes em atos infracionais está o baixo nível sócio-econômico. No entanto, vale ressaltar que não será apenas o fator pobreza que explicará as ocorrências de comportamento in- fratores, mas sim um conjunto de fatores de risco associados. Oliveira e Assis (1999), por exemplo, encontraram que regiões do Rio de Janeiro que apresentaram baixos indicadores sócio-econômicos não foram as áreas nas quais se encontraram as maiores taxas de mortes por homicídio.

Neste sentido, a pobreza seria um fator de risco ao fomentar um ambiente estressante, que ocasionaria problemas situacionais que, por sua vez, acarretariam prejuízos ao desenvolvimento humano (Barnett, 1997). Segundo Aiello e Willians (2000), caso apenas o fator econômico fosse levado em consideração, a desigualdade social no Brasil tornaria a população de crianças em situação de risco gigantesca.

Outro fator importante foi, além do baixo nível sócio-econômico, ter crescido em um ambiente composto por uma grande quantidade de integrantes familiares (Ozen et al., 2005). Além do fato da maioria das famílias dos menores em cumprimento de medida sócio-educativa de restrição de liberdade não ser nuclear, muitas delas tinham sofrido abandono de um dos pais, morte, bem como ocorrência de divórcio (Feijó \& Assis, 2005; Kierkus \& Hewitt, 2005; Ozen et al., 2005; Wong, 2011). Fenômeno este também observado por Galo e Williams (2005), ao verificarem que $43,1 \%$ dos adolescentes em conflito com a lei viviam apenas com a mãe. De fato, Feijó e Assis (2005) encontraram que a família dos adolescentes infratores não era nuclear em $78,7 \%$ dos casos estudados.

No entanto, Feijó e Assis (2005) destacam que não será apenas "famílias monoparentais" um fator determinante ao desajustamento no desenvolvimento infantil, mas sim a relação entre este fator com outras variáveis de risco, pois, no processo de reestruturação familiar de uma família nuclear para uma monoparental são necessárias reconfigurações de papéis entre os membros desta família, caracterizando assim uma fase de confusão emocional principalmente para as crianças que se encontram em processo de desenvolvimento.

A separação dos pais pode provocar, por exemplo, a ausência da figura paterna e assim privar os filhos de um modelo importante para 
formação de identidade social, o que pode acarretar em prejuízos ao desenvolvimento infantil. Neste sentido, não será a mudança estrutural em si que acarretará em prejuízos ao desenvolvimento infantil, mas sim as suas repercussões, tais como: dificuldades financeiras para cuidar dos filhos associadas à falta de apoio que podem acarretar no modo do estilo parental a ser desenvolvido (Galo \& Williams, 2005).

Dentre as condutas estabelecidas pelos pais, sabe-se que as famílias dos menores em cumprimento de medidas sócio-educativas com restrição de liberdade tiveram a experiência de violência familiar (Mack et al., 2007). Este fenômeno pode ser explicado segundo a teoria de modelação inicialmente proposta por Albert Bandura e Walters (1959), na qual a aprendizagem de comportamentos violentos depende da vivência de violência na vida cotidiana. Como defende Huesmann (1986), os roteiros adquiridos durante a infância serão utilizados como guias para o comportamento social e resolução de problemas no futuro, pois é função da família ter papel preponderante na educação dos filhos, assim como orientá-los para o desenvolvimento de suas potencialidades e para a direção no convívio social.

Existe ainda outro conceito que pode vir a explicar este fenômeno, a transgeracionalidade, a qual é entendida como algo que se sobrepõem aos componentes familiares, mantendo-se presente ao longo das gerações, reiterando a visão de repetição ou reprise de determinados processos familiares com diferentes particularidades em diversas situações e época de uma dada família. Sendo assim, a transgeracionalidade seria fator representativo dos processos que são repetidos pelas famílias de uma geração a outra, e por isso se mantêm presentes ao longo da história familiar (Wagner, 2005).

Segundo Lima et al. (2006), 92\% dos adolescentes infratores já haviam experimentado, pelo menos uma vez, algum tipo de violência no ambiente familiar. Dentre tal porcentagem, $89,6 \%$ foram violências materiais ou físicas. Mas não se pode esquecer que o abuso físico intrafamiliar é multidirecional, pois poderá ocorrer entre quaisquer de seus membros, por exemplo: pais e filho (a), irmãos, casal, entre outros. Esses eventos estressores vivenciados podem estar associados à manifestação dos comportamentos desadaptativos destas adolescentes e podem ser entendidos como facilitadores para a entrada na vida infracional (Grogan-Kaylor et al., 2008; Dell'Aglio, Santos, \& Borges, 2005).

No entanto, não se pode afirmar que ter sofrido violência familiar determina o comportamento delinqüente; não se deve vitimizar a criança duas vezes. Conforme afirmam Feijó e Assis (2005), estas famílias, por serem pressionadas pelas situações de vida diária e subjugadas pelas responsabilidades dos cuidados com as crianças, tendem a perder habilidades positivas de enfrentar a situação construtivamente e assim cair num padrão crescente de maus tratos por estarem isoladas do amparo social construtivo e do mundo que está além da sua comunidade de baixos recursos.

Além do que, embora seja responsabilidade dos pais ajudar os filhos no processo de crescimento sadio, na conquista de maturidade e da autonomia, tem que se considerar que não se pode desprezar os aspectos individuais do experienciador, pois até mesmo a trasgeracionalidade depende da interação do indivíduo com os componetes da sua família.

Tal descrição proposta anteriormente pode ainda explicar a relação entre os familiares em conflito com a lei e cometer infrações em amostras juvenis do sexo feminino e masculino. $\mathrm{Ou}$ seja, não seriam as características genéticas, como proposta em 1876 por Cesare Lombroso, que determinariam o comportamento infrator. Segundo Lombroso, haveria a existência do "criminoso nato", o qual apresentaria determinados sinais ou estigmas de natureza física e psíquica que o classificariam como uma espécie diferente de "homo sapiens", dando início à escola da “Criminologia Clínica” (Elbert, 2003).

Apenas um estudo, dos 19 encontrados, investigou um aspecto biológico como sendo um fator de risco ao desenvolvimento da conduta criminal dos adolescentes. No entanto, o referido trabalho vislumbrou a hipótese da existência de uma relação entre problemas gestacionais associados à conduta infratora futura, devido ao de- 
senvolvimento neurocognitivo do feto ser comprometido por causa da exposição de um fator externo à mãe, à droga cannabis na gestação, ou seja, nem neste único caso observou-se a visão de pré-determinações genéticas adquiridas entre gerações como propunha a criminologia clínica.

\section{Conclusão}

De acordo com a revisão dos artigos, pode-se constatar que não existe um fator de risco familiar único que determinará na ação de infrações à lei. Tampouco se observa que os atos de infração à lei serão explicados apenas por um fator sem levar em consideração a sua contextualização. Mesmo quando se levam em conta as características familiares, deve-se considerar que existe o encontro de diversos fatores de risco, pois, ao analisar os fatores de risco associados à família de forma individualizada, isto pouco revelará sobre a sua influência no processo de fomentação de comportamentos infratores. Apenas quando se contextualizam tais fatores e os relacionam entre si que se observa alguma relação entre as características familiares e os atos infracionais juvenis. Como, por exemplo, ao observar a estrutura familiar associada a fatores sócio-econômicos, pois não será a estrutura familiar que evocará situações de conflito com a lei, mas sim as dificuldades provindas dela, tais como: a dificuldade financeira que o grande número de membros em uma família pode gerar. Desta forma, comprova-se que este processo é multicausal, ou seja, será influenciado a partir da interação entre diversos fatores de risco.

Assim, por meio desta revisão, pode-se constatar uma quebra na visão antiga de que a prática infracional estava relacionada apenas com as estruturas familiares. No entanto, os fatores familiares têm sido considerados pela literatura científica um fator contribuinte para os processos criminógenos. Esta situação passa a preocupar muito as esferas sociais, pois este núcleo de convivência primária passa a ser considerado um fator de risco ao bem-estar de muitas crianças e adolescentes, ao invés de cumprir sua função de proteção aos mesmos, tornando aparente o quanto que a fragilidade dos vínculos familiares pode ocasionar às crianças e aos adolescentes comportamentos infracionais, por possibilitar a formação de desajustes em seu desenvolvimento humano.

Neste sentido, este estudo avança por possibilitar esclarecimentos desses fatores, e acrescentar que a eficácia de programas de redução à violência deve iniciar e perdurar durante a fase da infância e adolescência, precisando associar medidas que objetivem não só crianças/adolescentes em situação de risco social, como também nas suas famílias, assim como prioriza o estatuto da criança e do adolescente (ECA), pois falar de prevenção objetivando apenas o "adolescente infrator" ou com "desvio de comportamento" é uma lógica improdutiva e ineficaz que conduz ao erro de que a punição é a única forma de resolução do problema da violência juvenil.

Entretanto, sabe-se o presente trabalho apresenta limitações, pois existem outros descritores que podem ser utilizados para encontrar mais trabalhos sobre a presente temática. Muitos trabalhos infelizmente ainda se referem aos adolescentes em situações de conflito como a lei, como adolescentes delinquentes, infratores, etc., fato este que deixa à margem muitas pesquisas realizadas, bem como não foi feito uso de operadores boleanos e truncaturas que tornassem mais específica ou até mesmo abrangente a busca.

Outro fator que contribui para a limitação dos dados aqui apresentados é que as diversas pesquisas elencadas foram realizadas em diferentes países. Muitas foram realizadas no Brasil, Canadá, EUA, Holanda, México, Inglaterra, ou seja, as pesquisas apontam para resultados produzidos em diferentes contextos sociais e culturais. Neste sentido, os dados necessitam ser reinterpretados dentro do contexto brasileiro, haja vista que os próprios estudos apresentam as suas limitações particulares em generalizar os dados para sua região de estudo, quanto mais às demais localidades.

\section{Referências}

Aiello, A. L. R., \& Williams, L. C. A. (2000). O papel do inventário Portage na operacionalização em programas de educação precoce. Anais do $39^{\circ}$ Encontro das APAES do Paraná. Educação Especial: Para ser e compreender, 1, 22-35. 
Bandura, A., \& Walters, R. H. (1959). Adolescent aggression. New York: Ronald Press.

Barnett, D. (1997). The effects of early intervention on maltreating parents and theirs children. In $\mathrm{M}$. J. Guralnick (Ed.), The effectiveness of early intervention (pp. 147-170). Baltimore, MD: Paul H. Brookes.

Branco, M., \& Wagner, A. (2009). Os adolescentes infratores e o empobrecimento da rede social quando do retorno à comunidade. Ciência \& Saúde Coletiva, 14, 557-566.

Caldwell, R. M., Beutler, L. E., Ross, S. A., \& Silver, N. C. (2006). Brief Report: An examination of the relationships between parental monitoring, self-esteem and delinquency among Mexican American male adolescents. Journal of Adolescence, 29, 459-464.

Carter, H., Fortson, E. N., \& Dusten, R. H. (2006). The impacto $\mathrm{f}$ community disadvantage on the relationship between the family and juvenile crime. Journal of Research in Crime and Delinquency, 43, 326-356.

Carvalho, M. C. N., \& Gomide, P. I. C. (2005). Práticas educativas parentais em famílias de adolescentes em conflito com a lei. Estudos de Psicologia (Campinas), 22, 263-275.

Childs, K., Sullivan, C., \& Gulledge, L. (2011). Delinquent behavior across adolescence: Investigating the shifting salience of key criminological predictors. Deviant Behavior, 32, 64-100.

Christiansen, K., \& Knussmann, R. (1987). Androgen levels and components of aggressive behavior in men. Hormones and Behavior, 21, 170-180.

Davies, P. T., Myers, R. L., Cummings, E. M., \& Heindel, S. (1999). Adult conflict history and children's subsequent responses to conflict: An experimental test. Journal of Family Psychology, 13, 610-628.

Day, N. L., Leech, S. L., \& Goldschmidt, L. (2011). The effects of parental marijuana exposure on delinquent behaviors are mediated of neurocognitive functioning. Neurotoxicology and Teratology, 33, 129-136.

Dell'Aglio, D. D., Santos, S. S., \& Borges, J. L. (2004). Infração juvenil feminina: Uma trajetória de abandonos. Interação em Psicologia, 8, 191-198.

Elbert, C. A. (2003). Manual Básico de Criminologia (N. Fayet Jr., Trad.). Porto Alegre, RS: Ricardo Lenz.
Feijó, M. C., \& Assis, S. G. (2005). O contexto e de exclusão social e de vulnerabilidade de jovens infratores e suas famílias. Estudos em Psicologia_(Natal), 9, 157-166.

Galo, A. E., \& Willians, L. C. A. (2005). Adolescentes em conflito com a lei: Uma revisão dos fatores de risco para a conduta infracional. Revista Psicologia, Teoria e Prática, 7, 81-96.

Gold, J., Sullivan, M., \& Lewis, M. (2011). The relation between abuse and violent delinquency: The conversion of shame to blame in juvenile offenders. Child Abuse \& Neglect, 35, 459-467.

Grogan-Kaylor, A., Ruffolo, M., Ortega, R. M., \& Clarke, J. (2008). Behaviors of youth involved in the child welfare system. Child Abuse and Neglect, 32, 35-49.

Huesmann, L. R. (1986). Psychological processes promoting the relation between exposure to media violence and aggressive behavior by the viewer. Journal of Social Issues, 42, 125-39.

Kierkus, C. A., \& Hewitt, J. D. (2005). The contextual nature of the family structure/delinquency relationship. Journal of Criminal Justice, 37, 123-132.

Krischer, M. K., \& Sevecke, K. (2008). Early traumatization and psychopathy in female and male juvenile offenders. International Journal of Law and Psychiatry, 31, 253-262.

Lima, I. M. S. O., Alcântara, M. A. R., Almeida, K, V. D., \& Alves, V. S. (2009). Experiências de violência intrafamiliar entre adolescentes em conflito com a lei. Revista Brasileira de Crescimento e Desenvolvimento Humano, 16, 16-24.

Mack, K. Y., Leiber, M. J., Featherstone, R. A., \& Monserud, M. A. (2007). Reassessing the family-delinquency association: Do family type, family processes, and economic factors make a difference? Journal of Criminal Justice, 35, 5167.

Nardi, F. L., \& Dell Aglio, D. D. (2012). Adolescentes em conflito com a lei: Percepções sobre a família. Psicologia: Teoria e Pesquisa, 28, 181191.

Nijhof, K. S., de Kemp, R. A. T., \& Engels, R. C. M. E. (2009). Frequency and seriousness of parental offending and their impact on juvenile offending. Journal of Adolescence, 32, 893-908.

Oliveira, M. B., \& Assis, S. G. (1999). Os adolescentes infratores do Rio de Janeiro e as instituições 
que os "ressocializam": A perpetuação do descaso. Cadernos de Saúde Pública, 15, 831-844.

Ozen, S., Ece, A., Oto, R., Tirasci, Y., \& Goren, S. (2005). Juvenile delinquency in a developing country: A province example in Turkey. International Journal of Law and Psychiatry, 28, 430-341.

Pacheco, J. T. B., \& Hutz, C. S. (2009). Variáveis familiares preditoras do comportamento anti-social em adolescentes autores de atos infracionais. Psicologia: Teoria e Pesquisa, 25, 213-21.

Rodway, C., Norrington-Moore, V., While, D., Hunt, I. M., Flynn, S., Swinson, N., ...Shaw, J. (2011). A population-based study of juvenile perpetrators of homicide in England and Wales. Journal of Adolescence, 34, 19-28.

Secretaria Nacional de Promoção dos Direitos das Crianças e dos Adolescentes. (2011). Levantamento nacional do atendimento socioeducativo ao adolescente em conflito com a lei. Brasília, DF: Autor.

Tia, S., Merry, M., \& Suyeon, P. (2011). Late-adolescent delinquency: Risks and resilience for girls differing in risk at the start of adolescence. Youth e Society, 43, 1433-1458.
Wagner, A. (2005). Como se perpetua a família? A transmissão dos modelos familiares. Porto Alegre, RS: Editora da Pontifícia Universidade Católica do Rio Grande do Sul.

Webster-Stratton, C. (1998). Early intervention for families of preschool children with conduct problems. In M. J. Guralnick (Ed.), The effectiveness of early intervention (pp. 429-455). Baltimore, MD: Paul H. Brookes.

Wong, S. K. (2011). Youth crime and family disruption in Canadian municipalities: An adaptation of Shaw and McKay's social disorganization theory. International Journal of Law, Crime and Justice, 40, 100-114. 